\title{
Percepción de pacientes con enfermedad cardiopulmonar hacia la adherencia al ejercicio físico y las terapias complementarias
}

\section{Perception of patients with cardiopulmonary disease towards adherence to physical exercise and complementary therapies}

Jorge Salas-Cabrera ${ }^{1}$ iD ; Evanny Andrea Calvo-Zúñiga ${ }^{1}$ iD; Hibsen Alonso Jiménez-Rodríguez ${ }^{1}$; Diego Armando Fernández-Jiménez ${ }^{1}$ (iD)

\begin{abstract}
${ }^{1}$ Universidad Nacional de Costa Rica, Escuela Ciencias del Movimiento Humano y Calidad de Vida, Proyecto Rehabilitación Cardiopulmonar fase III, 2020. Jorge.salas.cabrera@una.cr; Vancy243@gmail.com; Hiljiro16@gmail.com;
\end{abstract} Diego.fdez.jinez@gmail.com

Cómo citar: Salas-Cabrera, J.; Calvo-Zúñiga, E.A.; Jiménez-Rodríguez, H.A.; Fernández-Jiménez, D.A. 2021. Percepción de pacientes con enfermedad cardiopulmonar hacia la adherencia al ejercicio físico y las terapias complementarias. Rev. Digit. Act. Fis. Deport. 7(2):e1934. http://doi.org/10.31910/rdafd.v7.n2.2021.1934

Artículo de acceso abierto publicado por Revista Digital: Actividad Física y Deporte, bajo una licencia Creative Commons CC BY-NC 4.0

Publicación oficial de la Universidad de Ciencias Aplicadas y Ambientales U.D.C.A, Institución de Educación Superior Acreditada de Alta Calidad por el Ministerio de Educación Nacional.

Recibido: marzo 1 de 2021 Aceptado: marzo 29 de 2021 Editado por: Álvaro José Gracia Díaz

\section{RESUMEN}

Introducción: Los tratamientos integrales y terapias complementarias en pro de la mejora de calidad de vida, incluye movimientos que contribuyen a mejorar el aparato músculo esquelético y respiratorio, estimula la coordinación, el equilibrio, la fuerza, además de crear beneficios sobre los problemas cardiovasculares. Objetivo: El propósito de este estudio fue evidenciar la percepción de pacientes con enfermedad cardiopulmonar hacia la adherencia al ejercicio físico y las terapias complementarias. Metodología: Se utilizó un enfoque cualitativo. Participaron 9 pacientes ( 2 mujeres y 7 hombres), la edad promedio de los pacientes es de 69,5 \pm 4 años, quienes pertenecen al proyecto de rehabilitación cardiopulmonar fase III, de la Escuela de Ciencias del Movimiento Humano de la Universidad Nacional. El peso promedio es de $76,57 \pm 2 \mathrm{~kg}$ y los problemas cardiacos, en general, son infarto MD, puentes coronarios y stents. Instrumento: Estos pacientes respondieron a una entrevista semiestructurada diseñada por el investigador y con validez de criterio de expertos. Se realizaron 5 preguntas comunes, acerca de aspectos relacionados a la percepción sobre adherencia al ejercicio físico y la realización de terapias complementarias. Análisis: Se realizó una triangulación de datos, utilizando las respuestas de los pacientes, aspectos relacionados a la teoría y al criterio del investigador, con base a lo revisado en la literatura. Resultados: Se puede determinar que la percepción de los pacientes respecto a la adherencia al ejercicio y a las terapias complementarias es muy buena y evidencias expresiones de satisfacción ante su ejecución, para mejora de la calidad de vida. Conclusión: Una estrategia eficaz para garantizar la adherencia al ejercicio en pacientes de un programa de rehabilitación cardiaca es la implementación de terapias alternas, que sean seguras y eficaz.

Palabras clave: Terapias complementarias; Adherencia al ejercicio físico; Percepción; Fase de mantenimiento o fase III.

\section{ABSTRACT}

Introduction: Comprehensive treatments and complementary therapies for quality of life improvement include movements that contribute to improving the skeletal and respiratory muscle 
system, stimulates coordination, balance, strength and creates benefits over cardiovascular problems. Objective: The purpose of this study was to show the perception of patients with cardio-pulmonary disease towards adherence to physical exercise and complementary therapies. Methodology: A qualitative approach was used 9 patients (2 women and 7 men) participated, the average age of patients is $69.5 \pm$ to 4 years, which belong to the phase III cardiopulmonary rehabilitation project of the School of Sciences of the Human Movement of the National University. The average weight is $76.57 \pm 2$ $\mathrm{kg}$ and the overall heart problems are MD infarction, coronary bridges and stents. Instrument: These patients responded to a semi-structured interview designed by the researcher and valid with valid expert judgment. Five questions were asked in general about aspects related to perception about adherence to physical exercise and the conduct of complementary therapies. Analysis: A triangulation of data was performed, using patient responses, aspects related to the theory and criterion of the researcher based on what was reviewed in the literature. Results: We can determine that patients' perception of adherence to exercise and complementary therapies is very good and evidences expressions of satisfaction in their execution to improve quality of life. Conclusion: An effective strategy to ensure adherence to exercise in patients in a cardiac rehabilitation program is the implementation of alternative therapies that are safe and effective.

Keywords: Complementary therapies; Adherence to physical exercise; Perception; Maintenance phase or phase III.

\section{INTRODUCCIÓN}

Según la Organización Mundial de la Salud (2018), Ryan et al. (2019), Metcalf et al. (2007) y Castillo et al. (2006), la principal causa de muerte en Costa Rica y en el mundo son las enfermedades cardiovasculares, seguidas por enfermedades pulmonares. Muchas son las condiciones que llevan a las personas a desarrollar problemas cardiovasculares, la inactividad física es uno de los principales factores de riesgo, que provoca el desarrollo de enfermedades no transmisibles, por lo tanto, la clave para reducir su morbilidad y mortalidad es promover actividades físicas, como estrategias de prevención, así como desarrollar programas de rehabilitación cardíaca, en donde los pacientes tengan la posibilidad de realizar ejercicio físico, reciban educación, atención psicológica y nutricional. Hoy en día es indispensable fomentar en los pacientes cardiacos, hábitos saludables, que coadyuven a mejorar su calidad de vida y fortaleciendo estrategias para lograr mayor adherencia hacia el ejercicio, como lo indican Franklin et al. (1998), Rivas (2011) y Petro (2010).

Un estudio realizado por Chabbar et al. (2019), concluye que el mayor beneficio de los programas de rehabilitación cardiaca fase II está basado, precisamente, en realizar cambios en los hábitos de vida saludables, que el paciente logra "aprender", mientras asiste a las terapias y la mejora significativa, que obtienen con respecto a la capacidad funcional; sin embargo, este mismo estudio, muestra una tendencia regresiva en cuanto a los valores de colesterol, de hemoglobina y de medidas antropométricas, que mejoraron significativamente durante el programa, pero que, lamentablemente, después de un año de finalizada la terapia, no se mantuvieron. Esta tendencia, se presenta en el momento, en que el paciente deja de tener contacto con los especialistas encargados de su cuidado; de ahí, la recomendación que los pacientes se mantengan asistiendo a todas las fases que ofrece los programas de rehabilitación cardíaca.

En este sentido, la fase de mantenimiento o fase III son esenciales para los pacientes, porque de acuerdo con Carballo et al. (2015) es una fase en donde se debe asegurar la continuidad del proceso asistencial, seguido de la persistencia en mantener y mejorar los hábitos de vida, alcanzados en la fase anterior $y$, con esto, lograr la adherencia hacia el ejercicio físico, debido a que la prevención secundaria, a largo plazo, representa un factor clave para el éxito de la calidad de vida de los pacientes, según Fernández et al. (2002) y Carballo et al. (2015); sin embargo, estudios recientes, muestran bajas adherencias al ejercicio físico en este tipo de población, tomando en cuenta que la adherencia, que Carballo et al. (2015) lo definen, como "el período de tiempo en el cual el comportamiento de una persona coincide con la recomendación médica o sanitaria".

Debido a lo anterior, García (2019) utilizó el cuestionario de Morisky-Green, para determinar pacientes adherentes y lo que consiguió no fue muy positivo, porque, un $72,55 \%$ de los pacientes del 
grupo control y un 52,08 \% del grupo de intervención, contestaron erróneamente. Por otra parte, Chabbar et al. (2019), un $48,7 \%$ de los pacientes, no cumplieron con el ejercicio físico recomendado y un $15,4 \%$ reconoció no realizar ninguna de las recomendaciones de ejercicio físico. Además, Carballo et al. (2015) muestra que solamente el $65,3 \%$ de los pacientes estudiados se encontraban realizando alguna actividad física. Incluso, Expósito et al. (2017) escriben que después de 6 años de terminado el programa de rehabilitación cardiaca, se pierde la capacidad funcional y menciona que los pacientes que siguen todas las recomendaciones pierden solo un $6 \%$ de su capacidad funcional, mientras que los pacientes que no tienen una buena adherencia, pierden hasta un $20 \%$ de su capacidad funcional.

Conforme a Zambrano et al. (2012), la falta de adherencia a los tratamientos farmacológicos y no farmacológicos de las enfermedades crónicas, continúa siendo un obstáculo para cumplir con el propósito de mejorar la salud y, con ello, la calidad de vida para muchos pacientes, lo que es preocupante, si se analiza junto con el estudio de Mejía (2020), donde hace referencia sobre la percepción de la calidad de vida en el adulto mayor y menciona que el $63,6 \%$, de un grupo de personas de 60 años y más, percibe la calidad de su vida como regular y tan solo el $36,3 \%$, lo percibe como buena; asimismo, alude que en la dimensión física, el 70,5\% de adultos mayores percibe la calidad de su vida como regular y solamente el $29,5 \%$, afirman percibir una buena calidad de vida.

El gran problema, de acuerdo con Andagoya (2017), radica en argumentar que, en la actualidad, hay muchos métodos de ejercicio físico, pero el principal problema es que no hay innovación por parte de los centros. En Costa Rica, desde hace dos décadas, se han implementado las terapias complementarias, como tratamientos integrales aliados a los de la medicina convencional, en el sistema de salud costarricense, como lo indican Anchía \& Revuelta (2017). Uno de los tratamientos integrales en pro de la mejora de calidad de vida y el déficit motor es el yoga. Para Bueno (2017), esta disciplina incluye movimientos que contribuyen a mejorar el aparato músculo esquelético y respiratorio, estimula la coordinación, el equilibrio, la fuerza, la flexibilidad y la respiración, además de crear beneficios sobre enfermedades físicas, como lo son problemas cardiovasculares, diversos tipos de cáncer, dolores crónicos de espalda y algunas disfunciones inmunológicas, según Lim \& Cheong (2015). Por tanto, como lo mencionan Zurita \& Vergara (2019), revolucionar en la aplicación de este tipo de técnicas es imprescindible, porque son actividades que ofrecen un estímulo complementario, a nivel físico y cognitivo, favoreciendo, en gran medida, la independencia de los adultos mayores.

Es por esto, que el propósito de esta investigación fue determinar, desde el enfoque cualitativo, la percepción que tienen los pacientes del proyecto de rehabilitación cardiopulmonar fase III de la Universidad Nacional de Costa Rica, donde se puso en práctica el ejercicio físico y las terapias complementarias, con el fin de conseguir la adherencia indispensable para mejorar la calidad de vida de los pacientes participantes del proyecto.

\section{MATERIALES Y MÉTODOS}

Participantes. Para este estudio cualitativo, se consideró un grupo de 9 pacientes (2 mujeres y 7 hombres), que pertenecen al programa de Rehabilitación Cardiaca Fase III de la escuela de Ciencias del Movimiento Humano, de la Universidad Nacional de Costa Rica, de los cuales, solamente 7 ( 2 mujeres y 5 hombres) participaron en la investigación. Al grupo de pacientes, se les pasó el consentimiento informado acerca de su libre participación en esta investigación y que podían elegir, si participaban o no, durante el proceso. La edad promedio de los pacientes es de 69,5 años, su altura promedio es de $1,69 \mathrm{~cm}$, su peso promedio es de $76,57 \mathrm{~kg}$ y los problemas cardíacos, en general, son Infarto MD, Puentes coronarios y Stents.

A los partícipes, se les aplicó una entrevista semiestructurada, para determinar su percepción a las terapias complementarias. Instrumentos y materiales: Con el fin de poder extraer la mayor cantidad de información correspondiente a la percepción que tienen los pacientes, en cuanto a la adherencia al ejercicio y al uso de terapias complementarias, se utilizó una entrevista semiestructurada, que fue elaborada por especialistas y se aplicó en las instalaciones de la Universidad Nacional, poco antes de iniciar una sesión de yoga. Análisis: Se procede a realizar una triangulación de datos, utilizando un enfoque cualitativo, en el cual, se va a triangular la entrevista semiestructurada, la teoría y el criterio de expertos en esta investigación. 


\section{RESULTADOS Y DISCUSIÓN}

Los resultados que se presentan a continuación son las expresiones más significativas que los usuarios comentaron posterior a la entrevista semiestructurada, realizada por el investigador. Las mismas fueron acomodadas de acuerdo a las dos grandes categorías de análisis.

\section{Adherencia al ejercicio:}

"...Se cambia la rutina y mientras haya ciertos elementos que le mantengan a uno el interés, uno va a continuar motivado..."

"...Lo importante es mantenerse lo más constante posible en el ejercicio..."

\author{
"...Esto tiene la parte de grupo social muy \\ importante..."
}

"...Se cambia la rutina y mientras haya ciertos elementos que le mantengan a uno el interés uno va a continuar motivado..."

"...Lo importante es mantenerse lo más constante posible en el ejercicio..."

\section{"...Esto tiene la parte de grupo social muy importante..."}

\section{Terapias complementarias:}

"...Son terapias que, así como la palabra lo dice, complementan lo que hemos venido haciendo. Es algo que adentro en el laboratorio no lo podemos hacer..."

“...Los ejercicios de respiración, como Taichi y Yoga, que son esenciales para el resto del cuerpo, en el siguiente orden, para estirar, para fortalecer la musculatura, para la flexibilidad, la tranquilidad del corazón y sacar un poco el estrés. Además de su fase espiritual..."

"...El yoga sí funciona como ejercicio, hace que uno se dé cuenta de ciertas habilidades, que uno tiene que mejorar y hace que se puedan ejercitar músculos, que regularmente no se usan..."

“...Con estas terapias es más agradable, más atractivo y uno no se desmotiva..."
La Real Academia Española (2018) define percepción, como "la capacidad para que a través de los sentidos se reciban impresiones o sensaciones externas, lo que también, implica que se pueda conocer algo o comprenderlo", por lo tanto, es solamente a partir de la experiencia vivida, que se puede determinar la percepción de una persona con respecto a algo. En este caso, la experiencia de este grupo de pacientes cardiópatas queda plasmada en el cuadro de resultados anteriores, donde se puede observar, a groso modo, que todos los comentarios han sido de carácter positivo. Solo viendo esto, se puede determinar que la percepción de estos pacientes con respecto a la adherencia al ejercicio y a las terapias complementarias, es muy buena; sin embargo, es necesario analizar más a fondo cada comentario y tomar en cuenta otros aspectos, como las observaciones de tipo cualitativas, que se han hecho a lo largo de todas las sesiones realizadas, para que, de esta manera, se pueda precisar lo que realmente concibe este grupo de pacientes, al incluirles las terapias complementarias, en sus rutinarios ejercicios.

Todos los participantes concuerdan en que el ejercicio físico se puede volver monótono, si no hay innovaciones o diferentes actividades dentro del programa; esto coincide con el estudio de Carballo et al. (2015), que hace referencia en que una de las limitaciones en cuanto a la adherencia a un programa de ejercicio en el anciano puede ser derivada, entre otras, como del aburrimiento.

También, los pacientes están conscientes de lo importante que es mantenerse constantemente practicando ejercicio físico; incluso, mencionan que mientras haya ciertos elementos que les mantengan el interés, como el caso concreto de las terapias complementarias, ellos continúan motivados a asistir al programa. Con esto es posible afirmar, tal como lo plantean López et al. (2017), que es un hecho que las personas buscan otro tipo de asistencia, que cubra sus necesidades de manera integral y están encontrando en las terapias complementarias las vías para conseguirlo.

A la hora de conversar con ellos, se puede notar el entusiasmo y el enardecimiento, cuando se refieren a cómo las terapias complementarias vinieron a cambiar la rutina y en cómo vinieron a convertir las sesiones más atractivas y entretenidas; los comentarios siempre son positivos, por ejemplo, uno de ellos expresó: "con estas terapias es más agradable, 
más atractivo y uno no se desmotiva". Acerca de esto, Anchía \& Revuelta (2017) coinciden en que los comentarios que se hacen con respecto a las terapias complementarias son siempre de carácter positivo, sea cual sea el tipo de terapia; tanto los pacientes como los profesionales encargados, continuamente, expresan palabras motivantes.

Es importante resaltar, que el tipo de terapias aplicadas a este grupo de pacientes fueron las terapias de mente-cuerpo que, como lo indican López et al. (2017), son técnicas que incrementan la capacidad de la mente para influir positivamente en las funciones corporales y dentro de ellas, están la meditación, el yoga, la musicoterapia, el Tai Chí, entre otras.

Para este estudio, en la mayoría de las sesiones, se practicó el yoga y cada viernes se podía percibir la emoción que cada uno de los pacientes tenía, porque iba a hacer algo diferente; el ambiente cambiaba, siempre son personas alegres, pero ese día, había algo diferente, la actitud era muy distinta y cambiaba totalmente el contexto.

Ellos comprenden que este tipo de terapias viene a integrar el trabajo físico que han estado haciendo, debido a los resultados esperados de la rehabilitación cardíaca que, tal como lo indican Acevedo et al. (2013), son el incremento de la capacidad aeróbica, mejora en la flexibilidad, resistencia y fuerza muscular, respuestas que son esenciales para el resto del cuerpo. El hecho de combinar estas ganancias con las terapias alternas, como el yoga, que es un medio, a través del cual, se puede controlar el estrés y brindarle mayor tranquilidad al corazón.

Se pudo conocer, que la mayoría de los estudios garantizan la eficiencia de la práctica de yoga, como una terapia alternativa específica, para la disminución de los niveles de estrés psicológico. Una revisión de Azofeifa (2018), escribe sobre su gran efectividad y menciona que alrededor del $75 \%$ de las personas que lo practican, presentan una disminución considerable del nivel de estrés. Además, según los resultados de todas las investigaciones analizadas muestran que la práctica del yoga, junto con el entrenamiento aeróbico es aún más efectiva.

Carballo et al. (2015), también mencionan que el ejercicio aeróbico puede reducir la tensión psíquica de los pacientes, modifican favorablemente la reactividad a determinados factores estresantes y que las personas que realizan ejercicios de forma regular refieren una sensación de bienestar relacionado con el mismo, que les ayuda a superar la sensación de depresión y ansiedad, que presentan con la enfermedad. Además, se ha demostrado, en estudios epidemiológicos, que la capacidad aeróbica también está ligada de forma inmersa y significativa a la mortalidad cardiovascular; basado en esto, se puede entender que se ha recomendado la práctica de actividad física regular, como estrategia para reducir incidentes, según Acevedo et al. (2013). La combinación del ejercicio aeróbico y el yoga en el adulto mayor juega un papel muy importante, porque les ayuda a obtener un bienestar integral y aún más, si se refiere a personas cardiópatas que, porsu condición, han sufrido mucho psicológicamente, debido a que, a raíz de su enfermedad, han experimentado un cambio radical en su vida, tanto en el ámbito laboral como familiar y social.

Para Carballo et al. 2015, la adherencia de los pacientes en programas de largo plazo o de fase III, representan un factor clave; el término adherencia puede ser entendido como el periodo de tiempo, en el cual, el comportamiento básico del paciente es igual o coincide con el tiempo establecido y recomendado medicamente. Con respecto a la adherencia al ejercicio físico, se han estudiado y sugerido métodos, para mejorar la adherencia a los programas de rehabilitación, para que se adquieran mayores hábitos cardiosaludables y que estos perduren; sin embargo, son muy pocos los trabajos existentes en la literatura médica sobre la adherencia a la fase III, como lo menciona García (2019).

Igualmente, García (2019) recalca que la adherencia requiere de un compromiso diario y que tiene múltiples barreras, incluida la motivación, porque, si no hay innovación con actividades diferentes que mantengan el interés, que hagan que el paciente perciba sensaciones positivas, la adherencia va a ser totalmente nula. Para Rocha et al. (2017), los objetivos de las fases de rehabilitación cardíaca buscan que los pacientes logren cambios en los comportamientos no saludables y, a su vez, promover las conductas saludables, como ejercicio físico constantemente y así, mantener estos hábitos, a lo largo del tiempo, más que todo, en las fases que, usualmente, no son supervisadas de manera permanente, en la que el paciente debe de mantenerse, por el resto de su vida, con cambios en actividades, para conservar la aptitud física y reducir los factores de riesgo. 
En el estudio de Carballo et al. (2015), se muestra una mayor adherencia al ejercicio físico, principalmente, en el grupo de 60-69 años, que puede ser comparable con este estudio, que muestra un grupo de pacientes, con una edad promedio de 69,5 años, donde todos concluyen que las terapias complementarias han venido a ser una extraordinaria herramienta, para mantenerse comprometidos con el ejercicio.

\section{CONCLUSIONES}

Los ejercicios implementados en un programa de rehabilitación cardíaca muestran sólidas mejoras, en cuanto a las capacidades funcionales y reducción de mortalidad cardiovascular y son fundamentales para la prevención secundaria, no solo confiriéndole sólidos beneficios, sino que también, generan un impacto positivo en la calidad de vida de las personas. La fase III de la rehabilitación cardíaca, se debe desarrollar a lo largo de la vida del paciente, en que se hace fundamental y primordial la corrección de factores de riesgo, asociados a posibles eventos cardiovasculares futuros y en la consolidación de cambios y modificaciones de los hábitos de vida, aprendidos durante el proceso en el programa.

El comportamiento adherente de los pacientes es complejo, debido a que, interactúan factores biológicos, conductuales, cognitivos y emocionales, que juegan un papel determinante a la hora de aplicar y seguir o no con los ejercicios. Esto implica dinamismo en las actividades, que fue el objetivo del presente artículo, determinar, desde el enfoque cualitativo, la percepción que tienen pacientes del proyecto de rehabilitación cardiopulmonar fase III, de la Universidad Nacional de Costa Rica, donde se puso en práctica el ejercicio físico y las terapias complementarias, con el fin de conseguir la adherencia indispensable, para mejorar la calidad de vida de los pacientes participantes del proyecto.

Es posible afirmar, que las terapias complementarias son una alternativa original y adecuada para mantener el interés y la motivación de los pacientes, con base en las respuestas obtenidas por cada persona, que dan aceptación y comodidad, a la hora de realizar los ejercicios, como el yoga, el Taichi, las Musicoterapias, entre otras. Por tanto, se concluye que una estrategia eficaz, para garantizar la adherencia al ejercicio en pacientes de un programa de rehabilitación cardíaca, es la implementación de terapias alternas, que sean seguras y eficaces, para que constituyan, junto a la rehabilitación cardiaca, un programa a largo plazo, que busca mejorar el estado fisiológico y psicosocial del paciente.

Agradecimientos. Un especial agradecimiento a la Universidad Nacional y a la Escuela de Ciencias del Movimiento Humano, por permitir que este manuscrito se pudiera realizar y a todos los usuarios participantes en dicha investigación, que permite la producción de nuevo conocimiento al mundo de la ciencia.

\section{REFERENCIAS}

1. ACEVEDO, M.; KRAMER, V.; BUSTAMANTE, M.; YÁÑEZ, F.; GUIDI, D.; CORBALÁN, R.; GODOY, I.; VERGARA, I.; JALIL, J.; FERNÁNDEZ, M. 2013. Rehabilitación cardiovascular y ejercicio en prevención secundaria. Revista Médica De Chile. 141(10):1307-1314.

2. ANCHÍA UMAÑA, I.; REVUELTA SÁNCHEZ, I. 2017. Terapias complementarias, salud y calidad de vida en Costa Rica. In 12ㅇ Congreso Argentino de Educación Física y Ciencias 13 al 17 de noviembre 2017 Ensenada, Argentina. Educación Física: construyendo nuevos espacios. Universidad Nacional de La Plata.16p.

3. ANDAGOYA, E. 2017. Guía de actividades lúdicas para mejorar la coordinación en niños con síndrome de Down en edades de $9-10$ años del Centro de Salud CRIEG de la ciudad de Guayaquil. Universidad de Guayaquil.

4. AZOFEIFA, C. 2018. Revisión de los beneficios de la intensidad y modalidades de ejercicio físico sobre el estrés psicológico. Pensar en Movimiento: Revista de ciencias del ejercicio y la salud. 16(1):1-21.

5. BUENO, D. 2017. Neurociencia. En: Bueno, D. Neurociencia. Octaedro - Rosa Sensat (España). p.11.

6. CARBALLO, R.; TÉLlEZ, Z.; GARCÍA, L.; MASSIP, J. 2015. Adherencia al ejercicio físico de los pacientes incorporados al programa de prevención y rehabilitación cardiaca. Revista Cubana de Medicina Física y Rehabilitación. $7(1): 25-41$. 
7. CASTILlO, L.; AlVARADO, A.; SÁNCHEZ, M. 2006. Enfermedad Cardiovascular en Costa Rica. Revista Costarricense de Salud Pública. 15(28):3-16.

8. CHABBAR, M.; CUKO, G.; GARZA, F.; ALBARRÁN, C.; BUSTAMANTE, E.; AMO, A. 2019. Prevención secundaria tras un síndrome coronario agudo. Resultados a medio plazo de un programa de rehabilitación cardiaca. Revista Colombiana de Cardiología. 26(5):264-271.

9. EXPÓSITO, J.; LÓPEZ, A.; AGUILERA, A.; VALLEJO, J.; PRAENA, J.; ECHAVARÍA, C. 2017. Impacto de la adherencia a las recomendaciones de hábitos de vida cardiosaludables en la capacidad funcional a largo plazo en pacientes que han completado un programa de rehabilitación cardíaca. Rehabilitación. 51(1):22-29.

10. FERNÁNDEZ, L.; SALINERO, M.A.; DEL RÍO, P. 2002. Prevención Secundario isquémica tipo ángor de miocardio en atención primaria. SEMERGEN - Medicina de Familia. 28(7):359-362. https:// doi.org/10.1016/S1138-3593(02)74086-0

11. FRANKLIN, B.; BONZHEIM, K.; GORDON, S. 1998. Rehabilitación del paciente cardíaco en el siglo XXI. Cambiando Paradigmas y percepciones. Revista de Actualización Ciencias del Deporte.

12. GARCíA, J. 2019. Eficacia de una internvención educativa en la adherencia al ejercicio físico de pacientes al finalizar un programa de Rehabiliatción Cardíaca. Universitat de Valencia.

13. LIM, S.; CHEONG, K. 2015. Regular Yoga Practice Improves Antioxidant Status, Immune Function, and Stress Hormone Releases in Young Healthy People: A Randomized, Double-Blind, Controlled Pilot Study. The Journal of Alternative and Complementary Medicine. 21(9):530538.

14. LÓPEZ RODRÍGUEZ, M.; ROMÁN LÓPEZ, P.; CORTÉS RODRÍGUEZ, A.E. 2017. Las técnicas complementarias. concepto y desarrollo. terapias complementarias en enfermería. En: Cortés Rodríguez, A.E.; López Rodríguez, M del M.; Román López, P. (eds). Técnicas complementarias en cuidados de enfermería.
15. MEJÍA, R. 2020. Calidad de vida percibida por los adultos mayores de un establecimiento de salud, Lima, Perú. Universidad Nacional Mayor de San Marcos.

16. METCALF, R.G.; JAMES, M.J.; GIBSON, R.A.; EDWARDS, J.R.M.; STUBBERFIELD, J.; STUKLIS, R.; ROBERTS-THOMSON, K.; YOUNG, G.D.; CLELAND, L.G. 2007. Effects of fish-oil supplementation on myocardial fatty acids in humans. Am J Clin Nutr. 85(5):1222-1228. https://doi.org/10.1093/ajcn/85.5.1222

17. OMS. 2018. Informe sobre la Actividad Física. Ginebra, Organización Mundial de la Salud.

18. PETRO, J. 2010. Beneficios de un programa de de rehabilitación cardiaca en la capacidad funcional y la calidad de vida relacionada a la salud con pacientes con cardiopatía coronaria. PublicE.

19. REAL ACADEMIA ESPAÑOLA. 2018. Diccionario de la lengua española, 23. a ed. Disponible desde Internet en: https://dle.rae.es

21. RIVAS, E. 2011. El ejercicio en la prevención de la rehabilitación cardiovascular. Science Direct Journal. 11(5):18-22.

20. ROCHA-NIETO, L.; HERRERA-DELGADO, C.; VARGAS-OLANO, M.O. 2017. Adherencia al tratamiento en rehabilitación cardíaca: diseño y validación de un programa de intervención biopsicosocial. Revista Colombiana de Psicología. 26(1):61-81. https://doi.org/10.15446/rcp.V26n1.53610

22. RYAN, J.; PETERSON, M.; RYAN, N.; SMITH, O.; O'CONNEL, N.; LIVERANI, S.; ANOKYE, N.; VÍCTOR, C.; ALLEN, E. 2019. Mortality due to cardiovasculardisease, respiratorydisease, and cancer in adults with cerebral. Developmental Medicine \& Child Neurology. 61(8):924-928.

23. ZAMBRANO, R.; DUITAMA, J.; POSADA, J.; JORGE, I.; FLÓREZ, J. 2012. Percepción de la adherencia a tratamientos en pacientes con factores de riesgo cardiovascular. Rev. Fac. Nac. Salud Pública. 30(2):163-174

24. ZURITA, K.; VERGARA, K. 2019. Beneficios del Hatha Yoga y la Neuroeducación del movimiento en los adultos mayores que asisten al programa de "Envejecimiento Activo" del Hospital Básico Durán. Universidad Católica de Santiago de Guayaquil. 